\title{
K-Means Clustering Algorithm based on Entity Resolution
}

\author{
B. Vinay Kumar \\ M.Tech.(SWE),Dept.of CSE \\ Kakatiya Institute of \\ Technology \& Science \\ Warangal-15,Telangana,India.
}

\author{
B. Raghu Ram \\ Assistant Professor, Dept.of \\ CSE Kakatiya Institute of \\ Technology \& Science \\ Warangal-15, Telangana, India
}

\author{
B. Hanmanthu \\ Assistant Professor,Dept.of \\ CSE \\ Kakatiya Institute of \\ Technology \& Science \\ Warangal-15,Telangana,India.
}

\begin{abstract}
Entity resolution is the problem of recognizing which entry in database refers to same cluster.in this we have to run the ER in order to reduce the running time and to obtain good results. This paper investigates how we can reduce the running of ER with minimum amount of work using $\mathrm{k}$-means clustering algorithm. In this, clustering can be done according to the matching of entries. We introduce a concept of technique called as k-means clustering to maximize the matching of entries identified using a limited amount of work. We illustrate the potential gains of this entity resolution approach using k-means.
\end{abstract}

\section{Keywords}

Data cleaning, Entity resolution-means Clustering Algorithm

\section{INTRODUCTION}

Entity resolution [1][2] is the problem of recognizing the entries that corresponds the real world entity. For ex if two companies want to combine their data the customer bought the product in one company and May also bought the different product in other company. Here the problem is that they will have the entries in two companies.in order to solve that problem the entries which are matched are identified and taken as single unit. Information integration is one of the most important and challenging problem in computer science. Information from sources must be combined so that users can access and manipulate the information in a unified way. One of the major problems in information integration is that of Entity Resolution. ER is a well-known problem that arises in so many applications.ER process is often extremely expensive due to very large data sets and compute-intensive record comparisons. We define our framework for how the k-means algorithm explains how it is fits in. In this Paper framework clustering based ER is defined. and also define a general model for Entity Resolution and then explanations is given on how the k-means clustering algorithm works on it.

\section{PREVIOUS WORK}

Implementation is the stage of the project when the theoretical design is turned out into a working system. Thus it can be considered to be the most critical stage in achieving a successful new system and in giving the user, confidence that the new system will work and be effective. The implementation stage involves careful planning, investigation of the existing system and it's constraints on implementation, designing of methods to achieve changeover and evaluation of changeover methods.

\subsection{Sorted List of Record Pairs}

In this module it gives a hint that consists of a list of entries pairs, ranked by the likelihood that the pair's match. Here in this ER algorithm uses either a distance or a match function. The distance function dry; so quantifies the differences between records $\mathrm{r}$ and $\mathrm{s}$ : the smaller the distance the more likely it is that $r$ and $s$ represent the same real- world entity. A match function matches so evaluates to true if it is deemed that $r$ and $s$ represent the same real-world entity. Note that a match function may use a distance function. For instance, the match function may be of the form "if dry; so $<\mathrm{T}$ and other conditions then true," where $\mathrm{T}$ is a threshold. If the distance between the two entries is so far we can know that the entries are so long that they do not match. if the distance between the entries are near we can say that they are nearer and preferred for matching. Here we use estimator which is less cost. If the distance between entries is small then they are said to be nearly matching.

\subsection{Hierarchy of Entry Partitions}

In this we give the partition of hierarchy as a possible format for the hierarchical clustering using k-means algorithm. This gives information likely on matching the entries in the form of partitions with the different levels of granularity where each possible partition represents a possible world ER. With the advantage of partition hierarchy it will reduce the storage space with a linear height. Here the clusters are splitted according to the entries if the entries are many the clusters are increased according to their matching's and entries. The ordering of entries are done according to their matching and comparing the entries and are given in same cluster. If there is more time we can start the comparison from starting in the higher levels of hierarchy.

\subsection{Ordered List of Record Pairs}

In this module it gives a hint that consists of a list of entries pairs, ranked by the likelihood that the pair's match. Here in this ER algorithm uses either a distance or a match function. The distance function dry; so quantifies the differences between records $\mathrm{r}$ and $\mathrm{s}$ : the smaller the distance the more likely it is that $r$ and $s$ represent the same real- world entity. A match function matches so evaluates to true if it is deemed that $r$ and $s$ represent the same real-world entity. Note that a match function may use a distance function. For instance, the match function may be of the form "if dry; so $<\mathrm{T}$ and other conditions then true," where $\mathrm{T}$ is a threshold. If the distance between the two entries is so far we can know that the entries are so long that they do not match. if the distance between the entries are near we can say that they are nearer and preferred for matching. Here we use estimator which is less cost. if the distance between entries is small then they are said to be nearly matching 


\subsection{K-means Algorithm}

The k-means criteria algorithm clusters tries to divide the entries in to groups of equal length decreasing a criteria called as inertia. this algorithm must have the numbers of clusters which we have to explain it has been used in large number of entries and large number of applications whatever the entry size it does not think about the entries. The main idea of this algorithm is used to divide the entries and arrange in the form of clusters in order to reduce the accessing time of entries. The k-mean algorithm divides the entries in to form of clusters and with the sets.in this algorithm it mainly focuses centroids on given entries. The main aim of k-mean algorithm is to choose the centroid in order to make inertia.in this algorithm it has three steps the first step is implementation of algorithm that choose the set of entries from the dataset. After initialization has the looping between the two other steps. In the first step it assign the entries in to nearest centroid value.in the second step it creates a new centroid by taking the mean values of all the centroid which are assigned to its previous one. The old values and new values are compared this process is repeated until the threshold value of the entries are reached and arranged in the form of clusters.it repeats until the entries are arranged without moving from its position. k-means are used for converging and minimum time is taken mainly comparison is done based upon the initiliasation of centroid as a result comparisons are done on several times in order to arrange the entries in to clusters this k-means algorithm has the centroids which are different from other initializations which give better results than random initialization what are done in another algorithms. Parallelization can be done by increasing the comparison cost. This algorithm can be done for $\mathrm{n}$ entries and for large values of datasets.Subsequent

\subsection{Entity Resolution Module}

In this model we take the set of inputs and run the ER process in order to take the output. The input is set of entries like e1, $\mathrm{e} 2, \mathrm{e} 3, \mathrm{e} 4$ in the each one entry is compared with another entry for the process of matching the entries with in the set of inputs. If the entries have matched with one another we take them as single unit and taken as one cluster .like this way the entries are matched by always running the ER process in order to reduce the space of a database.by putting them as single unit or cluster which are matching. If we want to search any entries in a database it will go directly to that cluster and search the entry whatth user want by doing this process the running time is reduced and the process of ER is shorten. We can get good results in the time overhead. This ER strategy works on similarity of the entries if the two entries have the same features they come on to one unit. Like this way a number of entries is given that many number of times comparison is done based on their similarity and the process of matching. The most pruning strategy is blocking or indexing [3][4][5][6] .inputs are kept in blocks according to their matching the entries are placed in to another blocks. For example sweet items have many items like chocolates and biscuits some company will follow their rules in order to placing the items in to their respective blocks.

\subsection{K-means Mini Batch Algorithm}

The mini k-Batch algorithm is a little bit different than the kmeans algorithm which uses mini batches in order to reduce the calculation time while it is showing the same objective function.In this algorithm it is randomly sampled based on the input set of data and it is sampled in each iteration in order to set the values according to their position in their clusters. These mini-batch algorithms drastically reduce the amount of calculation time required to converge to solution for the samples comparing with the other algorithm it reduces the convergence time of k-means algorithm. K-means generates results that are generally only slightly worse than the standard algorithm. The algorithms repeats between the two major steps in the first step it draws a samples from the given datasets in order to form a batch where this values are assigned to a nearest centroid.in the second step the given values to the centroids are updated. For our convenience this is done on sample basis and the given samples are formed in to batches and updated in to nearest centroid. By taking the continuous average of all the samples and previous samples assigned to that centroid. This has the effect of reducing the rate of change for a centroid over time. These steps are performed until limits of predetermined number of repetitions are reached.

\section{PROPOSED WORK}

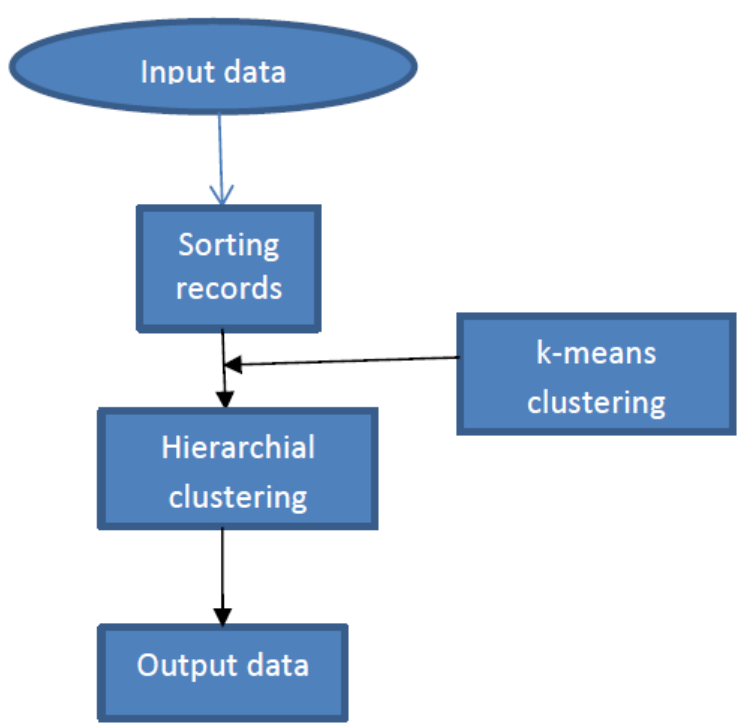

Fig.1 system architecture for k-means algorithm

In this architecture first the input of data is entered to it. Whatever the inputs are given the given inputs are sorted according to their list of pairs. Whatever the entries these are sorted according to lower or higher and higher to lower. The entries are ordered according to their matching's in to their respective clusters here we use k-means clustering algorithm In order to clustering of entries. k-means works as The kmeans algorithm clusters data by trying to separate samples in $\mathrm{n}$ groups of equal variance, minimizing a criterion known as the inertia or within-cluster sum-of-squares. This algorithm requires the number of clusters to be specified. It scales well to large number of samples and has been used across a large range of application areas in many different fields. The kmeans algorithm divides a set of samples and ordered according to their values by taking the centroids and arranges in the form of cluster.

\section{Ordered List of Records:}

In this module it gives a hint that consists of a list of entries pairs, ranked by the likelihood that the pair's match. Here in this ER algorithm uses either a distance or a match function. The distance function dry; so quantifies the differences between records $r$ and $s$ : the smaller the distance the more likely it is that $r$ and $s$ represent the same real- world entity. A match function matches so evaluates to true if it is deemed that $r$ and $s$ represent the same real-world entity. Note that a match function may use a distance function. For instance, the 
match function may be of the form "if dry; so $<\mathrm{T}$ and other conditions then true," where $\mathrm{T}$ is a threshold. If the distance between the two entries is so far we can know that the entries are so long that they do not match. if the distance between the entries are near we can say that they are nearer and preferred for matching. Here we use estimator which is less cost. if the distance between entries is small then they are said to be nearly matching

\section{Using application estimates:}

In this how we can conclude that it is matches is suppose for example the numbering is given to the students are according to their percentages. If the number is so closer then we can say that there is some matching in the terms of marks and conclude that they belong to one cluster

\section{Hierarchy of entry partitions:}

In this we give the partition of hierarchy as a possible format for the hierarchical clustering using k-means algorithm. This gives information likely on matching the entries in the form of partitions with the different levels of granularity where each possible partition represents a possible world ER. With the advantage of partition hierarchy we can reduce the storage space with a linear height. Here also we split the clusters according to the entries if the entries are many the clusters are increase according to their matching's and entries. The ordering of entries are done according to their matching and comparing the entries and are given in same cluster. If there is more time we can start the comparison from starting in the higher levels of hierarchy.

\section{K-means mini batch algorithm:}

The mini k-Batch algorithm is a little bit different than the kmeans algorithm which uses mini batches in order to reduce the calculation time while it is showing the same objective function.in this algorithm it is randomly sampled based on the input set of data and it is sampled in each iteration in order to set the values according to their position in their clusters. . These mini-batch algorithms drastically reduce the amount of calculation time required to converge to solution for the samples comparing with the other algorithm it reduces the convergence time of k-means algorithm. K-means generates results that are generally only slightly worse than the standard algorithm. The algorithms repeats between the two major steps in the first step it draws a samples from the given datasets in order to form a batch where this values are assigned to a nearest centroid.in the second step the given values to the centroids are updated. For our convenience this is done on sample basis and the given samples are formed in to batches and updated in to nearest centroid. By taking the continuous average of all the samples and previous samples assigned to that centroid. This has the effect of reducing the rate of change for a centroid over time. These steps are performed until limits of predetermined number of repetitions are reached.

\section{EVALUATION}

Frontend, the JAVA language was created by James Gosling in June 1991 for use in a set top box project. The language was initially called Oak, after an oak tree that stood outside Gosling's office - and also went by the name Green - and ended up later being renamed to Java, from a list of random words. Gosling's goals were to implement a virtual machine and a language that had a familiar $\mathrm{C} / \mathrm{C}++$ style of notation [9]. The first public implementation was Java 1.0 in 1995. It promised "Write Once, Run anywhere" (WORA), providing no-cost runtimes on popular platforms. It was fairly secure and its security was configurable, allowing network and file access to be restricted. Major web browsers soon incorporated the ability to run secure Java applets within web pages. Java quickly became popular. Backend Technology is MYSQL which is of Relational Database Management System and is Outcome of Open Source and Free Software. It is Free Widely used - Information Systems/embedded systems, primarily written in $\mathrm{C} / \mathrm{C}++$, available for Linux, Solaris, MS Windows and other Operating Systems. It is named after co-founder Monty Widenius's daughter, MymSQL- tweaks and hacks to form MySQL. MySQL AB, now a subsidiary of Sun Microsystems, which holds the copyright to most of the codebase " $\mathrm{AB}$ " part of the company name is the acronym for the Swedish "aktiebolag," or "stock company. The name of the MySQL Dolphin (MySQL logo) is "Sakila," which was chosen by the founders of MySQL AB from a huge list of names suggested by users in their "Name the Dolphin" contest. In this we used a front end as JAVA for coding the project and back end we use as SQL for storing the database in order to access the data for the project. In the home page it has records we have to give the random values for the records and also we can enter the values from back end directly to the database In the database the values are sorted according to their years and each year taken as single cluster Then in the front end it shows tables according to the years we have to give the number how many we want. It will calculate the distance and match function for years and gives the entity resolution and minimize the matrix to $1 * 1$ up to $n * n$ and next we have to start the entity resolution there it will find how many partitions we are given and the clustering is done to the entries according to the matching of entries which are taken from the database. In next page it will partition the records according to their matching values which are nearer to them and displays as one partition

The extension is the values we are taken as randomly. We should the know the units for the records whether the values entered are in kilograms, milliliter's So I took one column that if we give the unit as $\mathrm{ml}$ It will display all the ml column whether it is milk or oil something else In the example I took the records as bread,butter,ghee,milk

I gave the values in random as

$\begin{array}{lllll}\text { Bread } & \text { butter } & \text { ghee } & \text { milk } & \text { year } \\ 123 & 458 & 748 & 524 & 1991 \\ 232 & 784 & 756 & 125 & 1995 \\ 457 & 854 & 124 & 561 & 1991 \\ 412 & 745 & 785 & 321 & 1991\end{array}$

Inputted the values randomly It is sorted according to the year and I did the enhancement as these are given in values (numbers) I taken the units for these number for example milk is in $\mathrm{ml}$, butter is in gm. I gave the label as enter the units if I give $\mathrm{ml}$ in the textbox Output it will display all the records according to $\mathrm{ml}$ lists.

\section{K-means and ER algorithms}

For our experiments.in these paper three ER algorithms used to illustrate our k-means. In this section, we provide some implementation details for the ER algorithms used. For shopping records, B compares the titles, prices, and categories [7],[8]. For hotel records, B compares the states, cities, zip codes, and the names of the two hotels. We generate a pair list using minimum distance functions or from sampling. When generating pair lists using cheap minimum functions, we used 
the estimate function ear; so $1 / 4$ using the title (name) attributes of shopping (hotel) records as the sort key. Using e, we only Computed and stored the top Closest pairs to limit the time and space overhead. When using sampling to generate pair lists, we used a sample of 10 records. D measures the Jar distance between the titles of two records. For hotel records, D measures the Jar distance of the names of two records. We generate partition hierarchies in three ways:

i. Using sorted records,

ii. Hash functions,

iii. Sampling.

By default we set the number of levels of a partition hierarchy to 5. While increasing the number of levels helps us find more matching records early on, the benefits.

\section{CONCLUSION}

In the proposed work a k-means clustering algorithm approach for ER where given a limit in resources e.g. work runtime we attempt to make the maximum progress possible. We introduce the concept of K-means, which can guide an ER algorithm to Focus on resolving the more likely matching entries first. Our techniques are effective when there are either too many entries to resolve within a reasonable amount of time or when there is a time limit. We proposed three types of hints that are compatible with different ER algorithms: a sorted list of record pairs, a hierarchy of record partitions, and an ordered list of records after it gives a set of output clusters. We have also proposed various methods for ER algorithms to use these k-means. Our experimental results evaluated the overhead of constructing k-means as well as the runtime benefits for using k-means. We considered a variety of ER algorithms and two real-world data sets. The results suggest that the Benefits of using k-means can be well worth the overhead required for constructing.

\section{ACKNOWLEDGMENT}

Our thanks to the management members and principal of Kakatiya Institute of Technology and Science-Warangal who have facilitated resources to read and compute in order to develop this model and narrate this article and our sincere thanks to Head of the Department Prof.P.Niranjan who encouraged us research and publish this paper.

\section{REFERENCES}

[1] A.K. Elmagarmid, P.G. Ipeirotis, and V.S. Verykios, "Duplicate Record Detection: A Survey," IEEE Trans. Knowledge Data Eng., vol. 19, no. 1, pp. 1-16, Jan. 2007.

[2] A.K. Jain, M.N. Murty, and P.J. Flynn, "Data Clustering: A Review," ACM Computing Surveys, vol. 31, no. 3, pp. 264-323, 1999

[3] H.B. Newcombe and J.M. Kennedy, "Record Linkage: Making Maximum Use of the Discriminating Power of Identifying Information," Comm. ACM, vol. 5, no. 11 pp. 563-566, 1962.

[4] M.A. Herna'ndez and S.J. Stolfo, "The Merge/Purge Problem for Large Databases," Proc. ACM SIGMOD Int'l Conf. Management of Data, pp. 127-138, 1995.

[5] A.K. McCallum, K. Nigam, and L. Ungar, "Efficient Clustering of High-Dimensional Data Sets with Application to Reference Matching," Proc. ACM Sixth SIGKDD Int'l Conf. Knowledge Discovery and Data Mining, pp. 169-178, 2000.

[6] Gionis, P. Indyk, and R. Motwani, "Similarity Search in High Dimensions via Hashing," Proc. 25th Int'l Conf. Very Large Databases (VLDB), pp. 518-529, 1999.

[7] X.Dong, A.Y. Halevy, and J. Madhavan, "Reference Reconciliation in Complex Information Spaces," Proc. ACM SIGMOD Int'lConf.Management of Data, pp. 8596, 2005

[8] M. Weis and F. Naumann, "Detecting Duplicates in ComplexXML Data," Proc. 22nd Int'l Conf. Data Eng. (ICDE),p.109.2006. 